\title{
How to Celebrate Codd's RDBMS Vision
}

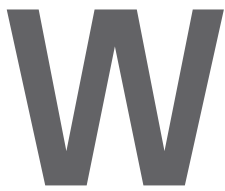

HILE WE WERE pleased Communications celebrated E.F. Codd's seminal article "A Relational Model of Data for Large Shared Data Banks" (June 1970) in "Happy Birthday, RDBMS!" by Gary Anthes (May 2010), we were also dismayed by its inaccuracies and misrepresentations, including about more than just pre-RDBMS history.

For example, saying "Codd's relational model stored data in rows and columns..." (emphasis added) is completely at odds with Codd's goal that "Future users of large data banks must be protected from having to know how data is organized in the machine." Rows and columns are the canonical representation of Codd's relations, not a constraint on physical data structures. Getting this wrong completely undermines Codd's contribution. Moreover, no viable commercial RDBMS has stored data purely in rows and columns, nor has any vendor completely implemented the logical and physical data independence his theory made possible.

Other inaccuracies and misleading statements abound:

DB2 did not "edge out IMS and IDMS." It took a long time for the transaction rates of any commercial RDBMS to compete with those of IMS, which remains an important commercial DBMS;

Ingres and its derivatives did not have the "DEC VAX market to themselves." Interbase, Oracle, and Rdb/VMS were early players (1980s), and Ingres was initially available on VAX/VMS butlike many RDBMS products that preceded the IBM products-introduced on Unix;

The "database wars" raged for almost two decades. Relational repeatedly had to prove itself against network, hierarchical, and object-oriented DBMSs, continuing with XML and Hadoop contenders;

Map/Reduce is a non-declarative programmer's distributed query template, and the Hadoop Distributed File System is a storage model. Neither rises to the level of data model or programming language;

Whether it was "easier to add the key features of OODBs to the relational model than start from scratch with a new paradigm" never happened. At best, features were added to SQL and SQLbased products, but these misguided additions did violence to the relational model's way of achieving desired capabilities, namely extensible domain support;

"Querying geographically distributed relational databases" is not unsolved. Implementing the relational model's physical data independence solved it;

Since 1980, numerous RDBMS products have provided partial implementation of physical data independence and been widely used in industry. Perhaps David DeWitt [cited by Anthes and director of Microsoft's Jim Gray Systems Laboratory at the University of Wisconsin-Madison] was referring to the problems of querying heterogeneous, distributed data with inadequate metadata, since he was quoted saying databases "created by different organizations" and "almost but not quite alike"; and

Database scalability has always been about numbers of concurrent users and locations, user variety, and manage ability, not just data volumes. One of us (McGoveran) published (late 1980s, 1990s) studies evaluating scalability of commercial products along these lines.

David McGoveran, Boulder Creek, CA

C.J. Date, Healdsburg, CA

\section{Author's Response:}

E.F. Codd's model let users "see" their data as if it were stored in ordinary tables, rows, and columns. This was easier for them to understand than the pointers and hierarchical trees used in other models. Such simplification was one reason the RDBMS model edged out IMS and IDMS, though IMS is still used in a few narrow (but important) niches. Alas, vendors did not implement Codd's rules in their purest form, as McGoveran and Date point out.

Gary Anthes, Arlington, VA

\section{Past Future Visions of Two-Way Cable Television}

The name "PLATO" in "Celebrating the Legacy of PLATO" by Kirk L. Kroeker (Aug. 2010) triggered my own memories from the early 1970s when I was researching a technology called two-way cable television, whereby interactive broadband services would be possible by blending computers and communications systems. I eventually published Talk-Back TV:Two-Way Cable Television (Tab Books, 1976), including an overview of the PLATO system.

I was reminded I had good things to say about the system, including about its plasma-panel display. But in considering PLATO as something that would work in a two-way-television environment, I suggested there would be a problem putting it onto a cable-television network because ordinary televisions could not do many things plasma panels could do. Leaving wiggle room, I added that PLATO researchers had produced considerable material that would work with ordinary CRTs.

Kroeker quoted Brian Dear saying PLATO was a computer system focused on connecting people and an excellent predictor of how the Internet would evolve. Maybe so, but the same could be said about technology being developed or envisioned as "two-way cable television" at the time.

In the same way an exploration of PLATO's history could "enrich everyone's overall perspective" of today's interactive, networked technologies, so, too, could a look back at visions of interactive broadband originally conjured 40 years ago.

Richard H. Veith, Port Murray, NJ

Communications welcomes your opinion. To submit a Letter to the Editor, please limit your comments to 500 words or less and send to letters@cacm.acm.org.

(C) 2010 ACM 0001-0782/10/1000 $\$ 10.00$ 